\title{
Beyond millennium development goals: a reality through life cycle interventions
}

\author{
H T Wickramasinghe ${ }^{1}$
}

Sri Lanka Journal of Child Health, 2009: 38: 49-53

Sri Lanka College of Paediatricians is still in its childhood, a product of the 43 year old Sri Lanka Paediatric Association. Veteran paediatricians of our country were all around at that time, nurturing this infant to grow so fast to an organization on par with any other college in the medical profession. The unwavering dedication of all the past presidents, council members and others behind the scene, have guided us thus far. I hope that future endeavours of this great institution will expand beyond the horizon while inspiring younger generations of paediatricians.

I have taken a different trajectory this time for the presidential address. My theme is "Beyond millennium development goals: a reality through life cycle interventions". In September 2000, at the dawn of a new millennium, at the United Nations (UN) millennium summit, world leaders agreed to a set of time bound and measurable goals and targets for combating poverty, hunger, disease, illiteracy, environmental degradation and discrimination against women. Placed at the heart of the global agenda, they are now called the millennium development goals ${ }^{1}$. I am concentrating only on the health indicators to show how far we have come and how we can improve our child care to go beyond those millennium goals without using much of the funds and resources most needed by our country today.

There are eight millennium development goals, set out by the UN to be achieved by year $2015^{1}$. My foremost concern is goal number 4 i.e. the reduction of child mortality ${ }^{1}$. It is very pertinent to know our present status with regard to all health indicators and the progress we have made so far. We have shown a dramatic and remarkable improvement of our health indicators since 1946. Our achievements in this respect

${ }^{1}$ Consultant Paediatrician, Colombo

* Address given on induction as President SLCP, September 2008.

are phenomenal, because these improvements have been achieved amidst social up-rising, political turmoil, economic constraints and never-ending terrorism. This and other indicators show that malnutrition and stunted growth of children still prevail at $24.5 \%{ }^{2}$. Fifty one percent of the population do not eat enough to satisfy minimum dietary requirements ${ }^{2}$. The latest figures of our infant mortality and mortality of children under 5 , show undeniable evidence that we have almost achieved what the United Nations wanted us to achieve in the year $2015^{2}$. Other health indicators such as maternal mortality, too, have shown similar decline ${ }^{2}$.

It is obvious that we cannot achieve this without a cost. It is therefore reasonable to examine how much we have spent to achieve these unbelievable results. Our budget is only $7.8 \%$ of the total government expenditure. This is in $2003^{3}$. Now it must be much less with the current situation prevailing in the country. Our expenditure is on par with all the other developing countries. In contrast, our health achievements are comparable to that of developed countries. The entire world has recognized our remarkable success and Save the Children Fund has released a booklet advising other nations to follow our health care policy.

Now our task ahead is very difficult. Firstly we have to maintain our achievements with limited resources. Secondly we have to show that we are going to go beyond millennium development goals. Let us now have a deeper look at the problem to see whether there are any spaces for improvement.

Although a good start in life begins well before birth, it is just before, during, and in the very first hours and days after birth that life is most at risk. Babies continue to be very vulnerable throughout their first week of life, after which their chances of survival improve markedly. 
All over the world, neonatal deaths takes major portions of the infant mortality especially in the developed and industrialized countries. Poorer nations still continue to have deaths after the first month of life due to infections and other communicable diseases. Neonatal deaths in our country constitute almost $80 \%$ of our total infant deaths ${ }^{4}$, surpassing even the most developed countries in the world. In Sri Lanka $44 \%$ of neonates die because of prematurity ${ }^{4}$. This is obviously the major cause of death in the developed countries as they have reduced the incidence of sepsis and birth asphyxia. However, just like in third world countries, we still have a major portion of neonatal deaths due to sepsis and birth asphyxia. Both these risk factors are preventable to a greater extent, thus allowing us some room for improvement of quality care. It is also important to remember that the majority of surviving babies of sepsis and birth asphyxia tend to be physically or mentally less able, increasing the burden on their parents and on their society. Thus, by reducing the incidences of sepsis and birth asphyxia alone, we could reduce our neonatal mortality so that we could stand as the only developing nation in the world showing health indicators almost on par with the developed world.

If you analyze the regional figures of all health indicators, what stands out clearly is that there are certain regions in the country except the war torn region showing persistently high mortality rates in comparison with the rest of the country.

I have identified three key issues from the available data.

1. Eighty percent of infant deaths occur during neonatal period.

2. Birth asphyxia and sepsis are still the leading preventable causes of neonatal deaths.

3. There is a very significant regional disparity in all health indicators, notably in provinces such as Central, North Central, and Sabaragamuwa, indicating a big flaw in the delivery of health service facilities in our country.

\section{What we can do?}

In developed countries, well-coordinated antenatal, intrapartum and postnatal care for mothers and newborns coincided with reduced rates of mortality before the introduction of neonatal intensive care in the early 1980s.

Intensive care facilities, specialists and expensive equipment are useful to reduce neonatal mortality even further only after very low levels have already been achieved. Rather than deploying high-tech instrumentation, we should work in line with the developed world by setting up a health care system with continuity of care between pregnancy, at birth, and care given when the mother is at home with her newborn.

Skilled professional care at birth is as critical for the newborn baby as it is for the mother. For example, effective midwifery ensures nontraumatic birth and reduces mortality and morbidity from birth asphyxia, while at the same time strict asepsis at delivery and cord care reduces the risk of infection. Skilled care makes it possible to resuscitate babies who cannot breathe at birth and to deal with or refer unpredictable complications as they happen to mother or baby. When the birth is appropriately managed by a skilled health worker, it is safer for both mother and newborn.

\section{What, then, are the problems?}

We need to identify the possible causes of failure on our part. Is it because of the illiteracy of mothers in these areas? Could it be a language barrier? In Sri Lanka majority of births occur in the hospital setting. In other countries, the weakest link in the care chain today is skilled attendance at birth. The main thrust of strategies aimed at improving the health of newborns should be to improve access to and uptake of professional care at birth by all pregnant women. I personally feel that the care given to the baby at home may be inappropriate owing to financial and social circumstances together with illiteracy and ignorance. This fragmentation of holistic care for newborns is not correctable at once. Educating parents and breaking their own social beliefs and cultural restrictions is no easy task. What is done before and at childbirth in the hospital setting should be linked with what will happen afterwards in the home and within the services that assume responsibility for providing health care for the newborn and, later, the child.

What we could do is to work together with other resources such as College of Obstetricians, Family Health Bureau, UNICEF and other 
responsible NGOs to formulate an inexpensive protocol to educate and update the knowledge and skills of health care providers i.e. local midwives, peripheral hospital doctors and other staff dealing with pregnancy, child birth and the postnatal period. At the same time, a strategy should be adopted to educate the public, particularly the recipient mothers in the region. This could be done via field programmes, posters, brief lectures at institutions etc. not forgetting the mass media. Media personnel are very important advocates of health care.

Millennium development goals are not the only health indicators of a country. There is a very important area we have forgotten. Life cycle interventions do not stop at antenatal, natal and postnatal care. It should be throughout life. This reminds me of Baker's hypothesis on fetal origins of adult disease. It is high time for us to explore the paediatrician's role in this respect. Following up this theory, by using 1911-1930 birth records for one English county (Hertfordshire), Barker showed that lower birth weight and weight at one year were associated with an increased risk of death from coronary heart disease and stroke.

Now new data is pouring in from various countries to show that type II diabetes, insulin resistance, hypertension and even metabolic syndrome and truncal obesity are all related to low birth weight. Low birth weight is a major issue in Sri Lanka almost amounting to $24 \%$ of live births ${ }^{3}$. Obviously low birth weight is a silent killer that no one has taken any notice of. Unlike prematurity, low birth weight is a preventable disease at least in some cases. Prevention of low birth weight should be on top of our priority list.

As paediatricians, we have a solemn duty to look after the children of our country. They are the future. If our duty is to look after their health only up to 12 or 15 years of age, then we are not doing justice to the future of our country. We should make sure, that the breeds we hand over to the future of this nation are healthy throughout their adulthood.

According to WHO, at least $80 \%$ of all cardiovascular disease and type 2 diabetes and over $40 \%$ of cancer could be avoided through a healthy diet, regular physical activity and avoidance of tobacco use ${ }^{5}$.
The problem of chronic diseases in adults in this part of Asia is now known to be in epidemic proportion and three causative factors stand out very positively viz. the genetic build up of South Asians, globalization and chronic westernization syndrome.

Genetic build of South Asians which presumably was a survival tool at times of famine in ancient times, is now, with abundance of food and lack of activity, behaving adversely on our health. In the present world today, what was life saving in the past, has become a death trap within our body.

\section{Why are we so susceptible to this?}

Our body composition has made us prone to many illnesses. Essentially, we have been handed the worst genetics of all time: small epicardial coronary arteries, high susceptibility for insulin resistance and metabolic syndrome, ischaemic heart disease, poor muscle mass (which in turn, reduces insulin utilization and sensitivity), high truncal fat deposition, and coexisting malnutrition.

Our bodies were designed to benefit from intense physical labour, and to sustain the climatic variations of the tropics in an agricultural society. Our physiology can better sustain famine, and better store energy than any other human population on the planet. Unfortunately, these very virtues in uncertain times have become the bane of our existence in modern civilization. Thus, when food sources are abundant, our bodies would store energy rather than use it. When our people emigrate to western societies and when our bodies are then bombarded with the energy rich foods high in fats, low in fibre and high in carbohydrates, our body's storage system is easily overwhelmed, and we inevitably develop fatty liver disease, metabolic syndrome and eventually diabetes. The diet intended for South Asians by nature is the very diet our ancestors consumed - rich in fibre, low in fat, and rich in complex carbohydrates with low glycaemic index. Unfortunately, very few of us, if any, consume the diet that our bodies were designed for. Instead, we hoard our innards with foods laden with refined carbohydrates and sugars, trans fats and indigestible fatty acid moieties. In a sense, we are slowly killing ourselves. 
By the same token, our bodies have not been designed for endurance. Scarcity of fast twitch fibres in our muscle mass is the reason for not winning any individual fast running event by any South Asian in any Olympic games in history.

What proof do we have to show that we are created to die from these chronic adult diseases?

The famous Pune study has clearly shown that we are born with high fat body composition for truncal obesity, high leptin and high insulin at birth already increasing insulin resistance ${ }^{6}$. Interestingly, a number of laboratories in the USA are studying genetic predispositions in South Asian populations. Several studies from the Brigham \& Women's Hospital in Boston have shown that South Asians have abundant polymorphisms that predispose them to altered metabolic physiologies. Over $40-50 \%$ of South Asians from India and Pakistan (and surely Sri Lanka) have a number of polymorphisms in the gene PPAR-gamma (Peroxisome proliferator activated receptor-gamma) which is the signaling pathway that potentiates insulin signaling in adipocytes $^{7}$. Many of us, who have reduced PPAR-gamma activity, have lowered insulin signaling, higher circulating sugar levels, and a genetic insulin resistance.

\section{But does this mean all hope is lost to us?}

Absolutely not! A number of studies are now beginning to show some prospects. For example, several studies have shown that frequent consumption of Omega-3 fatty acids, actually sensitizes PPARgamma activation and may reduce insulin resistance in those with this particular polymorphism.

Secondly globalization and urbanization have certainly changed the human life style to such an extent that obesity and obesity related adult diseases are now increasing alarmingly.

We, in Sri Lanka are now facing a dual class syndrome, i.e. in urban areas, obesity has become an epidemic whilst in rural areas, malnutrition is heading up as an epidemic. Both these extremes, particularly in childhood, influence harmfully escorting us to the same chronic diseases in late life. We have two contrasting enemies causing more or less the same fate to our future community. Elimination of malnutrition and elimination of obesity both need to be addressed on the same platform.

With rapid globalization, emergence of western life styles with fast food, outdoor eating habits, inactive leisure time activities such as watching TV and playing videos, have all impinged collectively and adversely on our health. This rapid, but concealed, global threat has eroded our society in such a way, that no one has ever realised that $80 \%$ of deaths in adults of our country are preventable, if we did not allow them to be scarf up by the effects of globalization and the so called "chronic westernization syndrome"

Let us now look at the effect of modern western diet on westerners. The recent atherosclerosis risk in communities study (ARIC study) on nearly 10,000 volunteers in North Carolina has shown that the present western diet is not suitable for even Americans ${ }^{8}$. The risk of having metabolic syndrome with even so called diet drinks is $34 \%$ higher than the control group.

Ladies and gentlemen, we have fought and are almost winning a battle against smoking, which has shown only a statistically significant association with lung cancer. Recent studies have shown that regular consumption of fizzy drinks for more than five years will increase carcinoma of the pancreas by $90 \%{ }^{9}$. Here you have a drink, which has a $90 \%$ risk of getting the world's most deadly disease, freely available everywhere including school canteens. Why are we sleeping? The fight against fizzy drinks will not be easy. Fizzy drink companies in Sri Lanka spend millions of rupees annually for advertisements alone. Who will support us in banning this menace?

What we have to realize is that our genetic buildup, lack of physical exercise, lack of parental interest or sometimes negative pressure from parents on participation of children in sports activities, non-availability of time for physical activities, excessive indulgence on watching TV and playing video games, excessive consumption of junk food and fizzy drinks are some of the positive contributory factors in modern children's life style, that would lead to chronic diseases in adulthood. Another important issue is that what is a prudent diet may not be the most suitable diet for us as our genetic build and other features are different. We ought to have our own culturally and socially acceptable diet, but most suitable to our high fat-low lean mass body. 
This leads me to another very important point. Research across the world in specific ethnic populations is showing that different racial groups have different metabolic physiologies. These effects have been appreciated particularly in the USA, where doctors have known for years the difference in response to antihypertensives across different populations. For example, black Americans do not respond to beta blockers as do whites, Hispanics, Asians etc. Similarly, South Asian bodies do not work in the same manner in which white American bodies were designed to work. The caveat that this raises is in the interpretation of clinical studies and trials performed in the USA and Europe. Most of what we know about the risk factors for heart disease - smoking, diabetes, insulin resistance etc., comes from the famous Framingham Heart Study conducted in the 1940s in the USA. The study population here was completely white. Thus, although most of the data does make sense and is applicable even to Sri Lankans, we must be very careful in taking any study that was done in a genetically different ethnic group consuming an entirely different diet and then applying that to our population.

The question then, obviously, is how do we make sense of all this. My opinion is very simple. Firstly, identify the risk factors that are commonly applicable across all populations and work to reduce them - smoking, exercise and healthy eating habits. Healthy eating habits for example, should include a diet that closely approximates our ancestral diets - high fibre, moderate/high complex carbohydrates with a low glycaemic index, and low in fats. Secondly, carry out prospective and randomized controlled trials within our populations, even with a relatively modest sample size powered to detect differences, to verify the conclusions of USA and European based trials. For example, is coconut oil bad for our physiology as it is considered bad for fair-skinned Europeans? Is the same high fat, low carbohydrate diet such as Atkins heralded for these populations applicable to us? Are the hypertension cutoffs and metabolic risk cutoffs in our populations the same as those for European populations? Without knowing the answer to any of these questions, it is impossible for us to know which risk factor reduction would benefit us most.

A task of this gigantic nature would cast doubts upon the reality of an achievement of such magnitude. The question of "can we do it" has an answer. Many countries have already done it. Developed countries like USA, Canada, Australia and UK have already shown $70 \%$ reduction in incidence of heart diseases ${ }^{5}$. If we slip in the right people as key role players into the scene, the task would be easy. Policy makers, decision makers, such as politicians, relevant ministries, civil societies, UNICEF, WHO and other NGOS, activists, consumer groups, journalists and mass media personnel, teachers, professors and other academics are some of these people.

The topics that should be incorporated into the planning should include:

- Prevention of IUGR and postnatal stunting.

- $\quad$ Breast feeding, weaning and infant nutrition

- Diet suitable for Sri Lankans : high fibre, fruits, vegetables and fish

- Exercise

- Avoidance of smoking

- Fish eating habit

- Mental exercise: meditation

- Lobbying against junk food advertisement

- School policies for junk food and fizzy drinks

To have a proper impact, it is very important to incorporate the basic identified key strategies to existing action plan programmes, so that there is no need for new funds. Safe motherhood programme of SLCOG, HSDP of SLCP for skills development in South and Uva provinces, public health forum of SLCCP, BFIN programme of FHB are all run by various colleges without proper coordination. Moreover, each of these programmes has many components of our substances for strategic planning. All what we need is to sit and discuss to come to a common platform with the same agenda.

Action plans are of two types. The comprehensive action plans need education of public. For that the support of media personnel, celebrities, and other public support groups are very crucial. We also have to identify some activists who will organize counter propaganda work for misleading advertisements etc. 
Integrated plans on the other hand need the support of other stakeholders such as Ministry of Education. National policy changes in relation to consumption of junk food and fizzy drinks and their proper implementation should be coordinated through a central body attached to the Ministry of Health.

What is most important to us today is to have policies and plans which could be implemented easily without using extra funds from the government. If we have the will, we can do this.

\section{References}

1. United Nations Development Programme: Millenium Development Goals. Available from: http://www.undp.org/mdg/basics.shtm

2. United Nations Millenium Development Indicators, July 2008 Data. Available from:

www.milleniumdevelopmentindicators. un.org/unsd/mdg/data.aspx

3. World Health Statistics 2008. Available from;

http:/www.who.int/whosis/whostat/200 8/en/index.html

4. Family Health Bureau-FA-UNICEF-U2007doc. Available from http://www.familyhealth.gov.lk/downlo de/FHB-FA-UNICEF-U-2007.pdf
5. Chronic disease and health promotion department sites. Available from: http://www.who.int/chp/topics/en/index.ht $\underline{\mathrm{ml}}$

6. Yajnik CS, Fall CHD, Coyaji KJ, Hirve SS, Rao S, Barker DJP, et al. Neonatal anthropometry: The thin-fat Indian baby. The Pune Maternal Nutrition Study. International Journal of Obesity 2003; 26:173-80.

7. Radha V, Vimaleswaran KS, Babu HNS, Abate N, Chandalia M, Satija P, et al. Role of genetic polymorphism peroxisome proliferator-activatedreceptorgamma2Pro12Ala on ethnic susceptibility to diabetes in South-Asian and Caucasian subjects: Evidence for heterogeneity. Diabetes Care 2006; 29: 1031-8.

8. Atherosclerosis Risk in Communities (ARIC) Study. Available from: $\underline{\text { http://www.cscc.unc.edu/aric/ }}$

9. Are Artificial Sweeteners the Answer? Available from: http://www.ecademy.com/node.php?id=9 $\underline{7499}$ 